\title{
Real-time assessment of platinum sensitivity of primary culture from a patient with ovarian cancer with extensive metastasis and the platinum sensitivity enhancing effect by metformin
}

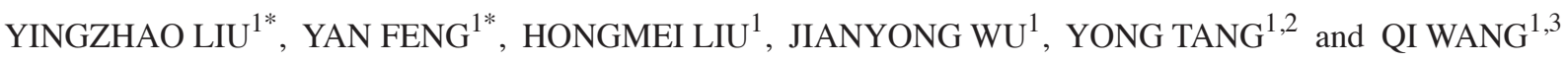 \\ ${ }^{1}$ Research Department, The Affiliated Tumor Hospital of Guangxi Medical University, Nanning, Guangxi Zhuang \\ Autonomous Region 530021; ${ }^{2}$ Urology Department, Wuming Hospital of Guangxi Medical University, Nanning, \\ Guangxi Zhuang Autonomous Region 530199; ${ }^{3}$ Key Laboratory of Early Prevention and Treatment for Regional High \\ Frequency Tumor, Ministry of Education, Nanning, Guangxi Zhuang Autonomous Region 530021, P.R. China
}

Received October 10, 2017; Accepted June 14, 2018

DOI: $10.3892 / \mathrm{ol} .2018 .9223$

\begin{abstract}
The aim of the present study was to perform a rapid evaluation of the efficiency of commonly used platinum-based chemotherapy regimens for patients with ovarian cancer with extensive metastases using an in vitro method combined with culturing primary cells and real-time monitoring, and to further explore the enhanced effect of metformin on susceptibility of ovarian cancer cells to platinum-based chemotherapy. The primary omental metastatic $(\mathrm{OM})$ cells were isolated from the omentum metastasis of a surgical patient with stage IIIc ovarian carcinoma. Drug sensitivity was evaluated using the xCELLigence system, and screening of the most effective platinum chemotherapy was performed through analysis of cell susceptibility to cisplatin, carboplatin, nedaplatin and paclitaxel or docetaxel alone or in combination. At the same time, this system was used to determine whether metformin was able to increase the sensitivity of cancer cells to platinum chemotherapy. The results revealed that nedaplatin exhibited the most marked cytotoxic effect on the OM cells, followed by those of carboplatin and cisplatin. The addition of docetaxel enhanced the cytotoxic effect, and the combination of platinum and paclitaxel also enhanced the effect. Metformin rapidly increased the sensitivity of cells to platinum-based
\end{abstract}

Correspondence to: Professor Qi Wang, Research Department, The Affiliated Tumor Hospital of Guangxi Medical University, 71 Hedi Road, Nanning, Guangxi Zhuang Autonomous Region 530021, P.R. China

E-mail: qi_catcat@163.com

Professor Yong Tang, Urology Department, Wuming Hospital of Guangxi Medical University, Nanning, Guangxi Zhuang Autonomous Region 530199, P.R. China

E-mail: cruisett@126.com

${ }^{*}$ Contributed equally

Key words: ovarian cancer, omentum metastases, primary cell culture, platinum, sensitivity, real-time cell analyzer system chemotherapy, and this effect was dose-dependent. The sensitivity of OM cells to different platinum-based regimens was varied. The effect of metformin on chemotherapeutic sensitization of cancer cells is clear in vitro, and the real-time cell analyzer assay has the potential to assist in determining individualized drug regimens for patients with metastatic ovarian cancer.

\section{Introduction}

Ovarian cancer has the highest mortality rate of all gynecological cancers worldwide and is frequently (in $>75 \%$ of cases) diagnosed at an advanced stage (1). Debulking surgery and platinum-based chemotherapy are, at present, the cornerstones of treatment (2). However, even with optimal debulking surgery followed by aggressive front-line chemotherapy, which results in an $80 \%$ initial cure rate, advanced-stage disease in the majority of cases is incurable (3). This is due to the development of chemoresistant disease, which results in recurrence within 16-22 months and a 5-year survival rate of only $27 \%$ (3). Therefore, the option of an effective platinum-based chemotherapeutic regimen to improve the sensitivity of primary platinum therapy to prevent tumor recurrence, extend the platinum-free interval and improve ovarian cancer survival rates has been the focus of clinical research.

The traditional approach to assess drug sensitivity in cancer cells includes the MTT cell viability assay, Cell Counting Kit- 8 proliferation assay, flow cytometry and adenosine triphosphate-based assays. However, these endpoint assays have similar disadvantages, such as multiple steps in their protocols that may lead to false-positive results, and steps performed at multiple time points to gain information during treatment, which may also introduce errors (4). To obtain accurate and uninterrupted information about the efficacy of drug combinations, the real-time cell analyzer system (RTCA) was selected for the present study. This system has been widely used to determine cell proliferation (4), evaluate drug sensitivity (5), screen drug profiles and reveal windows of drug response (6) in real time. The full list of references to 
the RTCA device is available via the ACEA Biosciences, Inc. website (aceabio.com/publications).

Metformin (MTF) has been used to treat type 2 diabetes mellitus for almost 60 years (7). In recent years, the effect of MTF on the treatment and prevention of cancer has become an important focus of research (8). MTF intake was reportedly associated with improved survival times in patients with ovarian cancer (9-11), and was also reported to increase the sensitivity of cells to cisplatin (DDP) in primary ovarian cancer (12). However, the mechanism of action was unclear and the potential benefit of MTF for patients without diabetes has not been confirmed.

In the present study, a patient that had received primary surgery who had not received adjuvant chemotherapy was selected to perform drug sensitivity testing. The aim was to establish a rapid method to identify the most effective chemotherapeutic combination and to determine whether MTF is beneficial to patients. Omental metastatic (OM) cells were isolated and cultured from a female patient with extensive invasive cancer. The RTCA system was used to evaluate the efficiency of common platinum-based chemotherapy regimens. In addition, this in vitro analysis also evaluated whether MTF could increase the sensitivity of platinum drugs.

\section{Patients and methods}

Patient. A 69-year-old female patient with ovarian cancer was hospitalized in the Gynecological Oncology department of The Affiliated Cancer Hospital of Guangxi Medical University (Nanning, China) in October 2016. The patient received a diagnosis of stage III ovarian cancer (FIGO staging system) (13) according to physical examination and diagnostic imaging tests and was scheduled for cytoreductive surgery. Written informed consent was obtained from the patient prior to surgery. The patient received no additional treatment prior to surgery, and was released from the hospital in December 2016. Postoperative pathology confirmed the specimen from the primary lesion was high-grade serous papillary carcinoma. The ethics review committee of The Affiliated Tumor Hospital of Guangxi Medical University approved the present study.

Chemicals. DDP and paclitaxel (PTX) were obtained from Hospira Australia Pty Ltd.; Pfizer Australia (West Ryde, New South Wales, Australia). Carboplatin (CBP) was obtained from Qilu Pharmaceutical Co., Ltd. (Shandong, China). Nedaplatin (NDP) was obtained from Jiangsu Aosai Kang Pharmaceutical Co., Ltd. (Jiangsu, China). Docetaxel (DTX) was obtained from Jiangsu Hengrui Medicine Co., Ltd. (Jiangsu, China). MTF hydrochloride was obtained from Hebei Tiancheng Pharmaceutical Co., Ltd. (Hebei, China). RMPI-1640 culture medium, fetal bovine serum, glutamate and $0.05 \%$ trypsin were obtained from Corning, Incorporated (Corning, NY, USA). Recombinant human insulin was obtained from Novo Nordisk (Bagsværd, Denmark).

Primary cell culture. Specimens from the transected primary lesions and OM cells were collected, cut into pieces and gently digested with $0.025 \%$ trypsin (cat. no. 25-053-CI; Corning Incorporated) in RMPI-1640 medium on a horizontal shaker for $15 \mathrm{~min}$ at $37^{\circ} \mathrm{C}$. Digested tissues were filtered with a 200-mesh filter. The unfiltered digested tissues were further crushed and filtered again through a 200 -mesh filter. The filtrate was collected and centrifuged at $300 \mathrm{x}$ g for $5 \mathrm{~min}$ at room temperature. The cells were resuspended in full culture medium consisting of RMPI-1640 medium, $20 \%$ fetal bovine serum, $1 \%$ glutamate, $0.01 \mathrm{mg} / \mathrm{ml}$ insulin, $100 \mathrm{U} / \mathrm{ml}$ penicillin and $100 \mathrm{U} / \mathrm{ml}$ streptomycin. The cells were then cultured $37^{\circ} \mathrm{C}$ in an incubator containing $5 \% \mathrm{CO}_{2}$. The phase-contract morphology of cells was observed with a magnification of x100 or x200 and recorded using an Olympus IX71 microscope (Olympus Corporation, Tokyo, Japan).

Cell viability and cytotoxicity assays on the RCTA platform. The cell viability and drug toxicity analyses were performed using the RTCA xCELLigence DP system (ACEA Biosciences, Inc., San Diego, CA, USA), a real-time and label-free system used to monitor cell viability, migration and invasion. For toxicity analysis, cells were plated in an E-Plate 16 culture plate of the RTCA system using full culture medium in $37^{\circ} \mathrm{C}$ overnight. Subsequently, the cells were monitored until the exponential phase, when they were treated with PTX (30 nM), DTX (50 nM), DDP (15 $\mu \mathrm{M})$, CBP $(330 \mu \mathrm{M})$, NDP $(95 \mu \mathrm{M})$, DDP $(15 \mu \mathrm{M})+\operatorname{PTX}(30 \mathrm{nM})$, CBP $(330 \mu \mathrm{M})+\operatorname{DTX}(50 \mathrm{nM})$, or NDP $(95 \mu \mathrm{M})+$ DTX $(50 \mathrm{nM})$, with or without $8 \mathrm{mM}$ MTF. For evaluation of effect of MTF, cells were treated with NDP $(95 \mu \mathrm{M})+\operatorname{DTX}(50 \mathrm{nM})$ with 4,8 and $16 \mathrm{mM}$ MTF. The viability and proliferation of the cells were monitored every $1 \mathrm{~min}$ in the first $2 \mathrm{~h}$ and monitored every $30 \mathrm{~min}$ for up to 200 h. Duplicate wells were used for each concentration of drug. The results are presented as the normalized cell index (CI), and were derived from the ratio of CIs prior to and following the addition of the compounds.

AlamarBlue $^{\circledR}$ cell viability assay. OM cells were plated at 2,500 cells/well in 96-well plates (Nalge Nunc International, Penfield, NY, USA). Detached and attached cells were counted using a Vi-CELL XR Cell Viability Analyzer (Beckman Coulter, Inc., Brea, CA, USA). For chemosensitivity assessment, $24 \mathrm{~h}$ after plating, cells were treated with PTX (30 nM), DTX $(50 \mathrm{nM}), \operatorname{DDP}(15 \mu \mathrm{M}), \mathrm{CBP}(330 \mu \mathrm{M})$ and NDP $(95 \mu \mathrm{M})$ only. Viability was assessed after 24, 36 and $48 \mathrm{~h}$ using an alamarBlue assay (Invitrogen; Thermo Fisher Scientific, Inc.; Waltham, MA, USA) according to the manufacturer's protocol, determined using Synergy microplate readers (BioTek Instruments, Inc., Winooski, VT, USA) with the fluorescence intensity at $590 \mathrm{~nm}$.

Western blot assay. Cells were pretreated with NDP at the indicated concentration alone or with MTF for $24 \mathrm{~h}$. Protein extracts were obtained using radioimmunoprecipitation lysis buffer (CW Biotech, Beijing, China) and protein concentration was quantified using a bicinchoninic acid protein assay kit (Thermo Fisher Scientific, Inc.), according to the manufacturer's protocol. Proteins $(40 \mu \mathrm{g})$ were separated by SDS-PAGE (10\% gels) and transferred onto polyvinylidene fluoride membranes (EMD Millipore, Billerica, MA, USA). Membranes were blocked with $5 \%$ bovine serum albumin in Tris-buffered saline containing $0.1 \%$ Tween-20 for $2 \mathrm{~h}$ at room temperature, and probed with antibodies against poly (ADP-ribose) polymerase, Rabbit anti-caspase 3 and GAPDH 

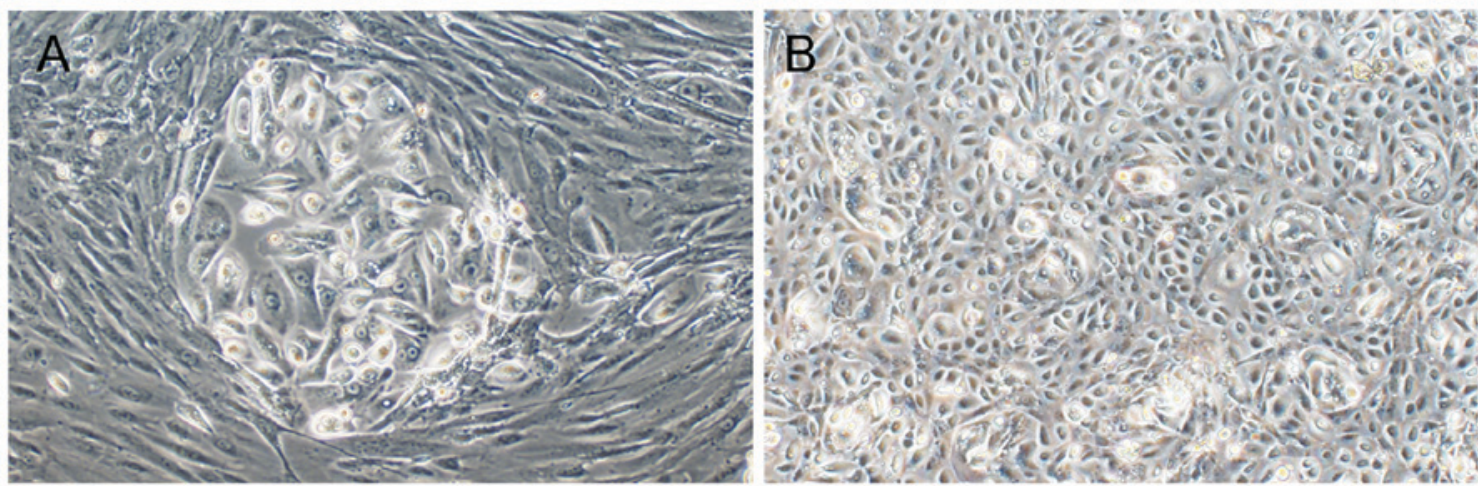

Figure 1. Morphology of ovarian cancer cells generated from the primary tumor and omentum metastases in a female patient aged 69 years with ovarian cancer who had extensive peritoneal metastasis. Typical phase-contrast images at day 4 are presented. (A) Ovarian cancer cells from the primary tumor; cancer cells were surrounded by fibroblasts (magnification, x200). (B) Cells generated from omental metastatic cells (magnification, x100).

at $4^{\circ} \mathrm{C}$ overnight. Rabbit anti-PARP (1:1,000; cat. no. 9542), Rabbit anti-caspase-3 (1:1,000; cat. no. 9665) and mouse anti-GAPDH monoclonal antibodies (1:1000; cat. no. 5174) were from Cell Signaling Technology, Inc. (Danvers, MA, USA). Then membranes were probed with secondary antibody for $2 \mathrm{~h}$ at room temperature. Secondary rabbit anti-mouse (1:3,000; cat. no. ab 6728) and goat anti-rabbit antibodies (1:3,000; cat. no. ab 6721) were from Abcam (Cambridge, MA, USA). Immunoreactive bands were visualized using the Pierce ECL Western Blotting Substrate (cat. no. 32106; Thermo Fisher Scientific, Inc., Waltham, MA, USA). Bands were scanned and analyzed using the FluorChem M system with AlphaView (version 3.4.0.0; ProteinSimple, San Jose, CA, USA). All experiments were performed three times.

In-cell ELISA assay. In total, 5,000 cells/well were seeded into a 96-well plate. The plates were incubated overnight at $37^{\circ} \mathrm{C}$ in $5 \% \mathrm{CO}_{2}$. Cells were treated with 24 or $48 \mathrm{mM}$ NDP with or without $8 \mathrm{mM}$ MTF. The expression of cleaved PARP or cleaved caspase-3 was analyzed using a human PARP (Cleaved) Multispecies In-Cell ELISA kit (cat. no. 62219; Thermo Fisher Scientific, Inc.) or human caspase-3 (cleaved) Multispecies In-Cell ELISA kit (cat. no. 62218; Thermo Fisher Scientific, Inc.) at 24 and $36 \mathrm{~h}$ after drug exposure. The results were normalized with the expression of $\alpha$-tubulin according to the manufacturer's instruction.

Statistical analysis. The CI readouts provided by the RTCA system are expressed as the mean \pm standard deviation from the duplicated results. Every result was from two repeats and in duplicated wells. Representative results are presented. A one-way analysis of variance with Bonferroni's post hoc test was used to compare the differences between groups. Statistical analyses were performed using SPSS for Windows (version 20.0; IBM Corp., Armonk, NY, USA). P<0.05 was considered to indicate a statistically significant difference.

\section{Results}

Primary ovarian tumor cells generated from the primary lesion and $O M$. In the present study, cancer cells were generated from the primary lesion and OM lesion successfully. The morphology of cells at day 4 is presented in Fig. 1. However, cells of different orientation had dissimilar morphology and biological characteristics. Cancer cells derived from the primary lesion were larger, polygonal, clustered and surrounded by fibroblasts (Fig. 1A). Following a number of passages, cells from the primary lesion lost the ability to proliferate and soon underwent apoptosis. The OM cells had an improved proliferative ability and were round and spaced apart from fibroblasts, and non-tumor cells were almost invisible (Fig. 1B). As the primary lesion had been removed, there was no requirement to further test the drug sensitivity in these cells. Thus, all cytotoxicity assays were conducted on Passage 3-Passage 5 OM cells.

Dynamic assessment of the cytotoxicity of commonly used chemotherapy drug combinations using RTCA. To avoid the drift of biological characteristics, cells used in the present study were limited to a maximum of five passages. At the time of experimentation, the cell morphology had not changed when observed under a microscope. The commonly used chemotherapy drug combinations tested in the present study were selected as advised by the National Comprehensive Cancer Network 2014 clinical practice guidelines for ovarian cancer (https://www.nccn.org/patients/guidelines/quick_guides/ ovarian/treatment_planning/index.html) and the evaluation of the physician in charge of treatment.

DDP, CBP and NDP were the most commonly used platinum-based drugs for the treatment of ovarian cancer (14). The sensitivity of OM cells to these drugs alone was initially analyzed. Platinum drugs combined with PTX or DTX are standard treatments for ovarian cancer (14). Thus, the cytotoxicity of the chemotherapy drug combinations, including DDP+PTX, CBP+DTX, and NDP+DTX, were also assessed (Fig. 2).

Initially, to rapidly screen the most effective drug, single and combination regimens were tested at certain concentrations calculated according to the dosage based on body surface area. The biosensors detected the attachment of OM cells and began to produce impedance-based readout values of $\mathrm{CI}$ at 4-5 $\mathrm{h}$ after plating. Cells entered the exponential phase of proliferation at $\sim 5 \mathrm{~h}$ after plating. The cells were then exposed PTX (30 nM), DTX (50 nM), DDP $(15 \mu \mathrm{M})$, CBP $(330 \mu \mathrm{M})$ or NDP $(95 \mu \mathrm{M})$ when the CI value reached 0.9 to 10 (Fig. 2A).

As presented in Fig. 2, non-treated OM cells (control) maintained aggressive proliferation. Neither PTX or DTX was 

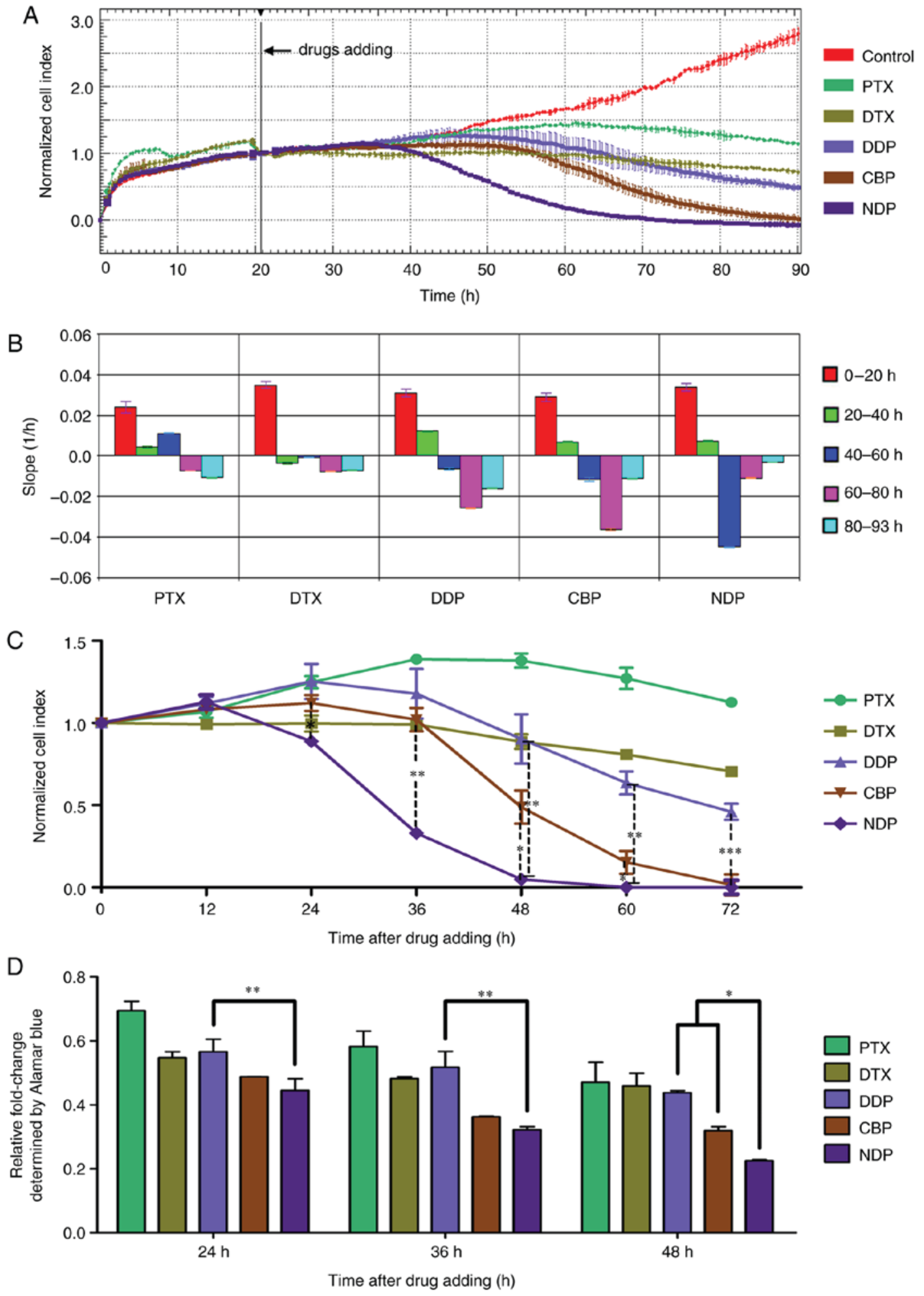

Figure 2. Real-time cell analysis of the cytotoxic effect of single chemotherapy drugs on OM cells. OM cells were seeded into the E-Plate and the CI was determined. (A) The normalized CI of OM cells treated with PTX $(30 \mathrm{nM})$, DTX $(50 \mathrm{nM})$, DDP $(15 \mu \mathrm{M})$, CBP $(330 \mu \mathrm{M})$ or NDP $(95 \mu \mathrm{M})$. The drugs were added at $\sim 20 \mathrm{~h}$ as indicated by the black line. The normalized CI was generated by normalizing CIs to the CI at the time of drug addition. (B) Slopes of CI curves in (A) at 0-20 (pretreatment), 20-40, 40-60 and 60-80 h. (C) The normalized CI was calculated at various time points. The results are expressed as the mean \pm SD. ${ }^{*} \mathrm{P}<0.05,{ }^{* *} \mathrm{P}<0.01$ and ${ }^{* * * *} \mathrm{P}<0.001$, compared with DDP; (D) The inhibitory rate of single chemotherapy drugs on OM cells was calculated on the basis of cell viability analyzed using an alamarBlue assay. The results are expressed as the mean \pm SD. ${ }^{*} \mathrm{P}<0.05,{ }^{* *} \mathrm{P}<0.01$. OM, omental metastatic; CI, cell index; CBP, carboplatin; PTX, paclitaxel; DDP, cisplatin; DTX, docetaxel; NDP, nedaplatin; SD, standard deviation.

able to induce apoptosis of OM cells as indicated by their CI curves, which became flat following exposure to PTX or DTX (Fig. 2A); the slope was close to zero (Fig. 2B), suggesting that those drugs only inhibited the proliferation of OM cells.
The cells exposed to DDP, CBP, and NDP all exhibited a typical downward shift in their proliferation curves (Fig. 2A). The earliest and sharpest decline was observed following NDP treatment, indicating the start of apoptosis at $20 \mathrm{~h}$ after 
the addition of drugs, whereas those treated with CBP and DDP exhibited a more gradual decline in their proliferation curves and apoptosis commenced at $35 \mathrm{~h}$ after the addition of drugs (Fig. 2A). The most extensive apoptosis occurred at $\sim 30 \mathrm{~h}$ following exposure to NDP, and at $\sim 50 \mathrm{~h}$ following exposure to CBP or DDP. The slope of the CI value exhibited the same trend (Fig. 2B). The CI declined to almost 0 at 40 and $70 \mathrm{~h}$ after exposure to NDP or CBP. However, the CI curve for the OM cells treated with DDP reached a plateau $70 \mathrm{~h}$ after treatment, and the CI value only declined to $\sim 60 \%$, which may indicate that these OM cells were less sensitive to DDP. The cell viability normalized from CI indicated that, from $24 \mathrm{~h}$, NDP had a statistically significant killing effect compared with DDP and CBP $(\mathrm{P}<0.05)$. This difference existed up to $72 \mathrm{~h}$ (Fig. 2C). To confirm this result, a chemosensitivity assay was conducted using alamarBlue. The results revealed similar trends, in that NDP exhibited the strongest toxicity towards OM cells, followed by CBP and DDP (Fig. 2D).

The present study demonstrated that combinations of platinum with taxanes were more toxic to OM cells compared with platinum alone. The CI curves began to gradually decrease following the addition of the chemotherapeutic drugs except DDP+PTX, compared with the slight increase in cells treated with platinum only (Fig. 3A). The slopes of each drug combination between 0 and $20 \mathrm{~h}$ exposure (Fig. 3B) were similar to those of PTX or DTX alone (Fig. 2B), and indicated that this result may be the effect of the taxanes. The normalized CI curves revealed that NDP in combination with DTX exhibited the most cytotoxicity among drug combinations without MTF in OM cells (Fig. 3A). The slopes also indicated that the steepest decline occurred at $\sim 20$ to $40 \mathrm{~h}$ after exposure to NDP+DTX. The steepest decrease occurred between 40 and $60 \mathrm{~h}$ after treatment with CBP+DTX or DDP+PTX (Fig. 3B and C, C-1). However, the CI curve of cells treated with DDP+PTX, as well as DDP alone, entered a plateau $70 \mathrm{~h}$ after treatment (Fig. 3A). The combination of NDP and DTX exhibited the most marked killing ability in the three combinations between 24 and $72 \mathrm{~h}$ $(\mathrm{P}<0.01$; Fig. 3C, $\mathrm{C}-1)$.

MTF increases drug sensitivity in cells in a dose-dependent manner. It has been reported previously that MTF improves ovarian cancer cell sensitivity to platinum-based drugs (10). In the present study, the effect of MTF on OM cells was further examined. As hypothesized, the CI curve declined more and the slopes were steeper during the first $20 \mathrm{~h}$ of exposure, and the time it took for CI to approach zero was decreased in every combination with MTF compared with the treatments without MTF (Fig. 3A and C, C2-C4). In DDP+PTX+MTF-treated cells, the CI fell below 0.2 with MTF, compared with 0.4 without MTF, at $70 \mathrm{~h}$ after treatment. Between 24 and $72 \mathrm{~h}$, in different combinations, adding MTF in all groups led to an increased cytotoxic effect $(\mathrm{P}<0.01$; Fig. 3C, C2-C4). Furthermore, the combination of NDP+DTX with MTF was examined at 4,8 and $16 \mathrm{mM}$. The results demonstrated that MTF improved the cell sensitivity at 12, 24 and $36 \mathrm{~h}$ $(\mathrm{P}<0.05$; Fig. 4).

MTF increases platinum drug-induced apoptosis. To further confirm that the decrease in the CI value was the result of apoptosis, PARP expression was analyzed by western blotting. MTF alone was not able to induce apoptosis; however, the apoptosis induced by NDP was enhanced as the PARP expression decreased along with the dosage of NDP when MTF was used (Fig. 5A-C). The expression of caspase-3 was also increased (Fig. 5A-C). To establish whether PARP or caspase-3 were activated, an in-cell ELISA was performed and it confirmed that MTF at concentrations as low as $8 \mathrm{mM}$ was able to significantly $(\mathrm{P}<0.05)$ increase the amount of cleaved PARP and cleaved caspase-3 (Fig. 5D and E).

\section{Discussion}

Ovarian cancer is the most lethal gynecological malignancy affecting women worldwide and the fifth most common cause of cancer-associated mortality among women in the USA (15). According to statistics, only $45 \%$ of patients with ovarian cancer have a survival time of 5 years following their diagnosis (16). In recent decades, despite the development of novel therapeutic strategies, surgery and platinum-based chemotherapy remain the standard treatments for ovarian cancer (17). The clinical chemotherapy options are limited, as patient reactions to the chemotherapy vary and chemoresistance develops rapidly, particularly for patients at the middle and advanced stage of ovarian cancer. The majority of patients with ovarian cancer have their primary ovarian tumors removed and subsequently succumb to metastatic disease rather than their primary tumor (18). In the present study, the patient OM-derived primary cells were investigated for chemotherapy drug sensitivity using the xCELLigence system. The results demonstrated that the metastatic cell sensitivity to the commonly used clinical chemotherapy drugs varied considerably. The cell responses to certain drugs, such as NDP and DTX, were positive; however, for other drugs, such as CBP and PTX, the cytotoxic effects were not evident. The results of the present study also revealed that MTF exhibited a promising sensitization effect and may assist in reversing drug resistance.

Pelvic metastases are common in ovarian cancer (19). Currently, the treatment of pelvic metastatic ovarian cancer relies on platinum-based chemotherapy regimens $(1,2,19)$. The clinical selection of drugs for these patients often relies on doctors' experience and lacks clear drug markers $(2,19)$. However, the majority of patients eventually become resistant to chemotherapeutic drugs and this causes the prognosis of these patients to be unsatisfactory (20). Previous studies for ovarian cancer were primarily based on cells generated from primary lesions $(21,22)$, and few of these studies focused on the omentum (23). It has been suggested that metastatic cancer is a markedly heterogeneous disease at the genetic, transcriptomic and microenvironment levels (24). Therefore, the results generated from primary lesions could not fully reflect the properties of metastasis. In addition, the heterogeneity between tumors indicated that drug sensitivity may also vary for each patient. A rapid method to determine the sensitivity to the drug was beneficial for effective drug regimen selection. In the present study, primary tumor cells were successfully isolated and cultured from the patient's omentum, and the platinum sensitivity was evaluated by the xCELLigence system within 10 days of surgery. 

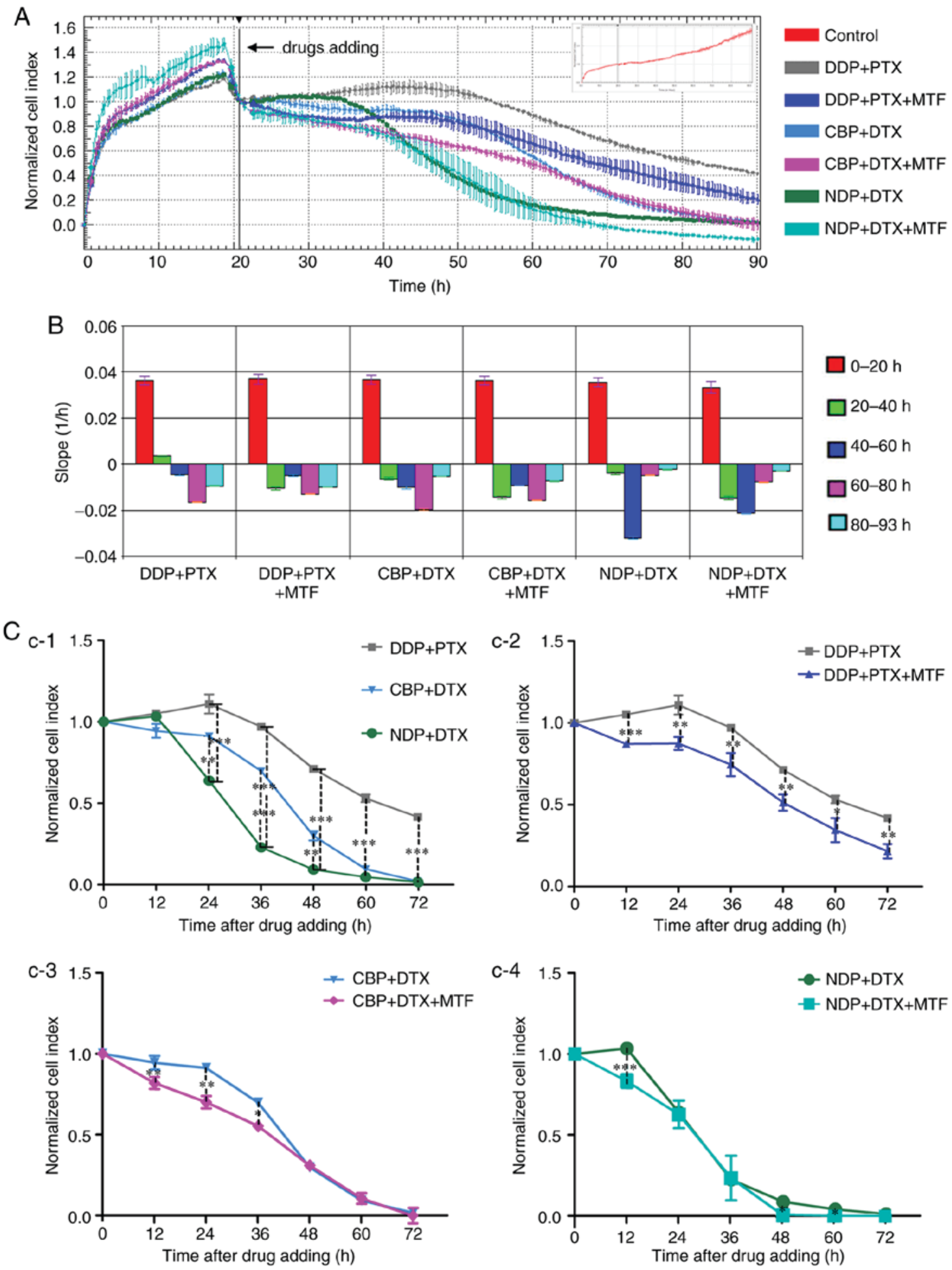

Figure 3. Real-time cell analysis of the cytotoxic effect of chemotherapy drug combinations on the OM cells. OM cells were seeded into the E-Plate and the CI was recorded. (A) The normalized CI of OM cells treated with DDP $(15 \mu \mathrm{M})+\mathrm{PTX}(30 \mathrm{nM}), \mathrm{CBP}(330 \mu \mathrm{M})+\mathrm{DTX},(50 \mathrm{nM})$, or NDP $(95 \mu \mathrm{M})+\mathrm{DTX}(50 \mathrm{nM})$, with or without $8 \mathrm{mM}$ MTF. The drugs were added at $\sim 20 \mathrm{~h}$ as indicated by the black line. The normalized CI was generated by normalizing CIs to the CI at the time of drug addition. The CI of non-treated cells is presented in the inset as the CI reached 3.0 at the end of experiment. (B) The slope of CI curves in (A) at 0-20 (pretreatment), 20-40, 40-60, 60-80 and 80-93 h. (C) The normalized CI following the addition of drugs were calculated (C-1: DDP+PTX vs. CBP+DTX vs. NDP+DTX; C-2: DDP+PTX vs. DDP+PTX+MTF; C-3: CBP+DTX vs. CBP+DTX+MTF; C-4: NDP+DTX vs. NDP+DTX+MTF). The results are expressed as the mean \pm standard deviation. ${ }^{*} \mathrm{P}<0.05,{ }^{* * *} \mathrm{P}<0.01$ and ${ }^{* * * *} \mathrm{P}<0.001 ; \mathrm{C}-1$, compared with DDP+PTX; C-2 to C-4, compared with drug combinations without MTF respectively. OM, omental metastatic; CI, cell index; CBP, carboplatin; PTX, paclitaxel; DDP, cisplatin; DTX, docetaxel; NDP, nedaplatin; MTF, metformin.

The response of the cells to different chemical drugs was varied, which could not have been predicted by clinical experience. Certain first-line chemotherapeutics, such as CBP, did not produce a satisfactory effect even at a high concentration
(330 $\mu \mathrm{M})$. Compared with other primary cultured cell lines, primary omentum ovarian cancer cells in this case were resistant to DDP. This indicated that this tumor cell type was not effectively killed by this widely used chemotherapy regimen, 
A
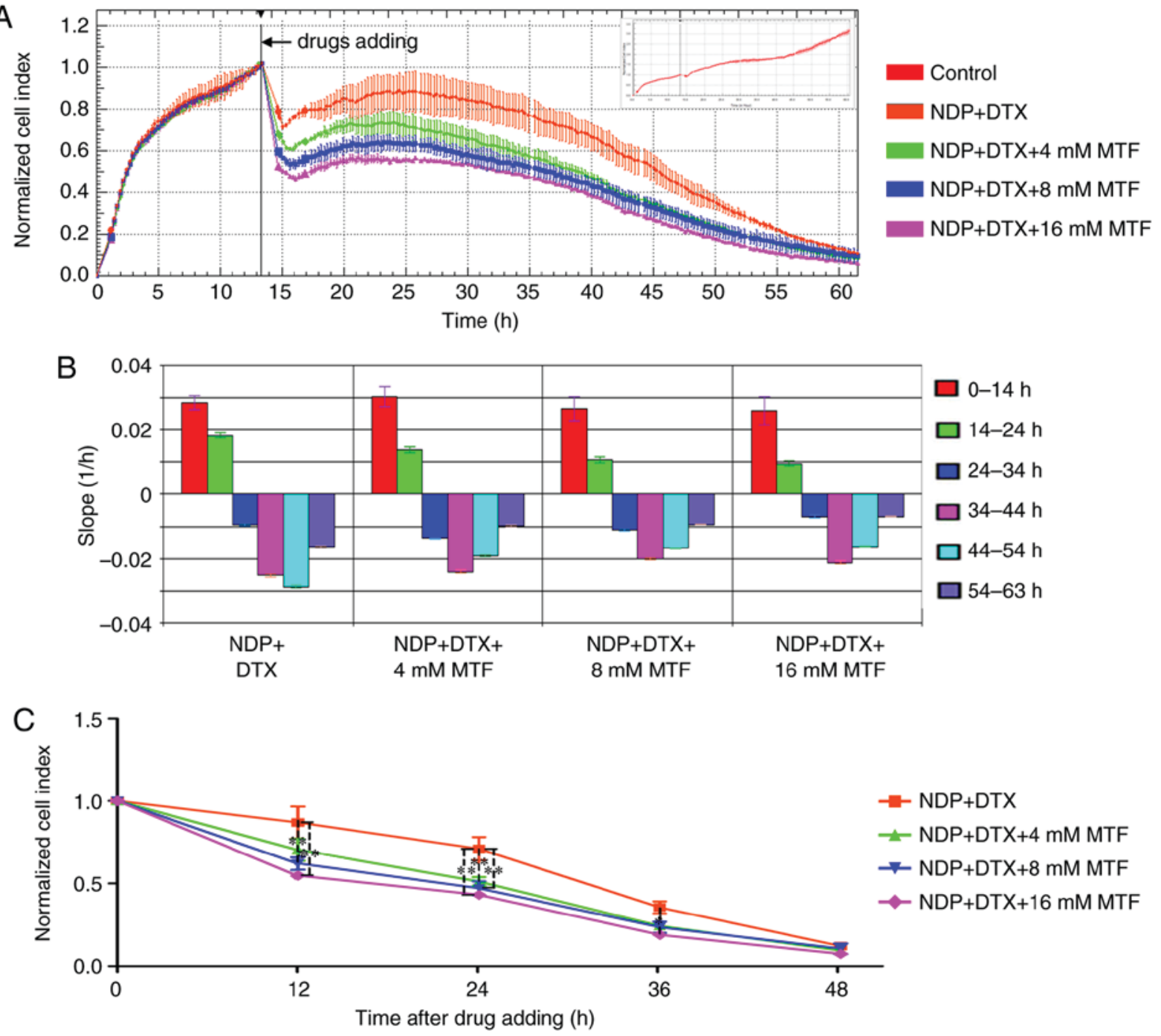

Figure 4. Real-time cell analysis demonstrates that the chemotherapy sensitization effect of MTF was dose-dependent. In total, 5,000 OM cells were seeded into the E-Plate and the CI was recorded. (A) The normalized CI of OM cells treated with NDP $(95 \mu \mathrm{M})+\mathrm{DTX}(50 \mathrm{nM})$ with 4,8 and 16 mM MTF. The drugs were added at $\sim 14 \mathrm{~h}$ as indicated by the black line. The normalized $\mathrm{CI}$ was generated by normalizing CIs to the CI at the time of drug addition. The $\mathrm{CI}$ of non-treated cells is presented in the inset as the CI reached 3.5 at the end of the experiment. (B) The slope of CI curves in (A) at 0-14 (pre-treatment), 14-24, 24-34, 34-44, 44-54 and 54-63 h. (C) The normalized CI was calculated at various time points. The results are expressed as the mean \pm standard deviation. ${ }^{*} \mathrm{P}<0.05$ and ${ }^{* *} \mathrm{P}<0.01$. OM, omental metastatic; CI, cell index; DTX, docetaxel; NDP, nedaplatin; MTF, metformin.

and drug-resistant cells may become the principal reason for tumor recurrence. In clinical practice, if it were possible to tailor individualized treatment based on the specific patient response to different treatments, the curative effect would be improved.

The resistance of ovarian cancer to platinum-based treatment may be intrinsic or acquired, and is brought about through a wide array of mechanisms (25). This includes pumps that eject the drug from the cell (26), to promoting the expression of genes that enable alternative growth pathways $(27,28)$, as cancer cells explore all avenues in their bid to survive and proliferate (25). Further complicating matters is the time spent understanding which mechanism or mechanisms are active in any particular individual (29). It is hoped that the results of drug susceptibility testing are useful in platinum-based chemotherapy to help achieve optimal chemotherapeutic effects, or at least to increase treatment intervals and improve the quality of life of the patient.

There were limitations to the present study, such as the primary cells used were a mixture of fibroblasts or other cell types, even though fibroblasts almost disappeared within a few passages. Conversely, fibroblasts were able to supply cytokines and maintain the in vivo microenvironments for tumor cells. In the present study, only cells between P3 and P5 were used when the fibroblasts were almost invisible. Furthermore, the fetal bovine serum used for cell culture may be replaced by an improved serum-free formula to minimize the influence of the cytokines in serum.

MTF, the most widely used drug for type 2 diabetes, has advantages including fewer side effects and low cost (7). It has been demonstrated that MTF effectively inhibits the proliferation of numerous ovarian cancer cell lines, including DDP and taxol chemoresistant cell lines (30). Previous clinical studies and in vitro analysis indicated a promising application of MTF to improve the effect of chemotherapy for ovarian cancer $(9,10)$. In the present study, the enhanced sensitivity of MTF was confirmed in OM cells from one patient without diabetes. Western blot analysis demonstrated that MTF could accelerate apoptosis of OM cells caused by NDP. 


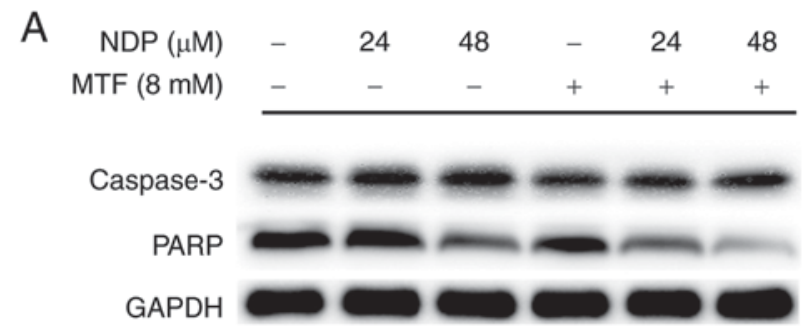

B
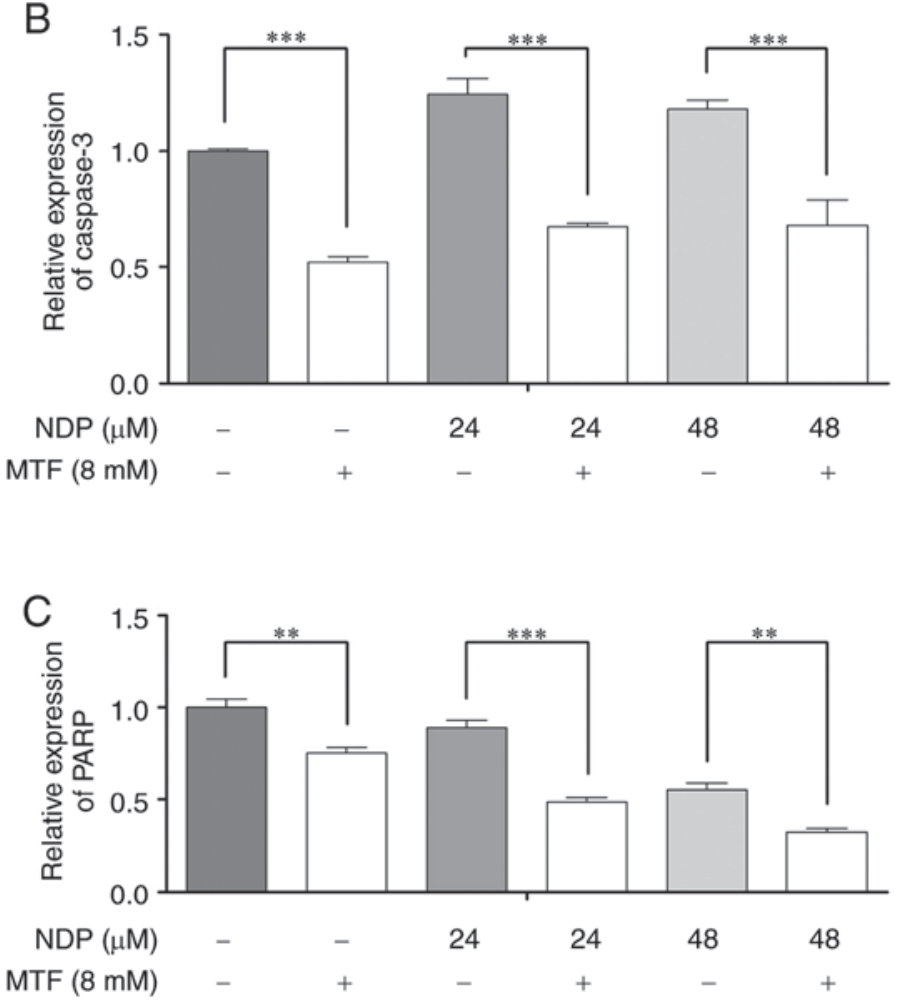
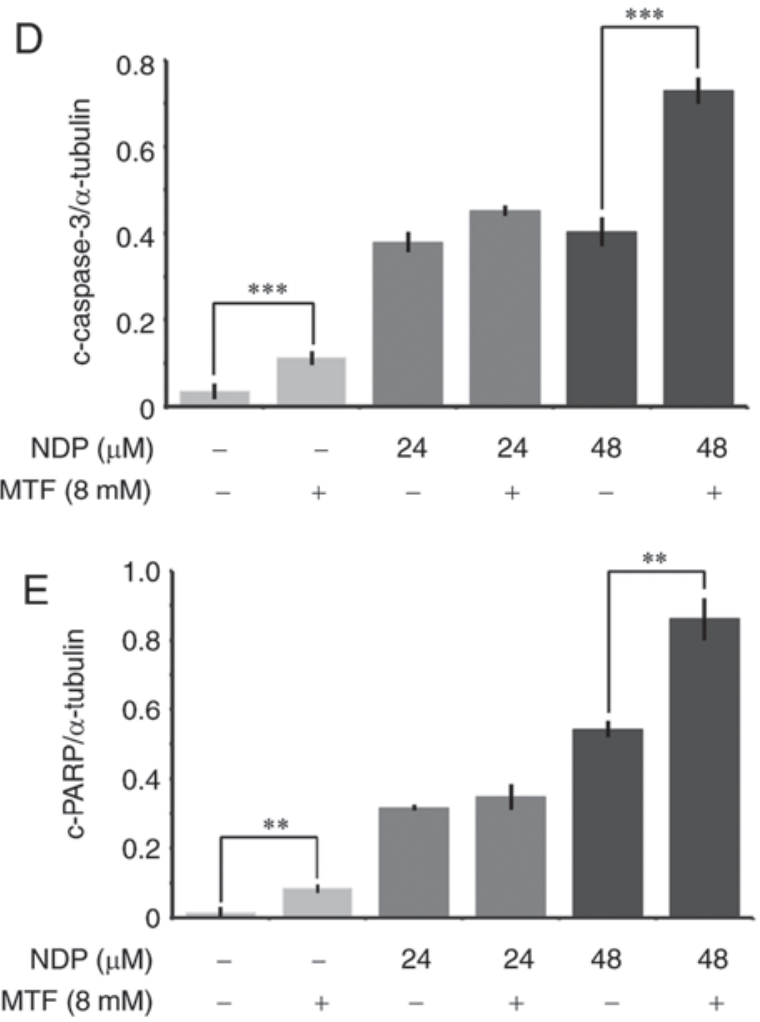

Figure 5. MTF-enhanced NDP induces apoptosis. (A) MTF increased the caspase-3 and PARP expression in omental metastatic cells. GAPDH was used as the loading control. Relative cell expression of (B) caspase-3 and (C) PARP. (D) Cleaved caspase-3 and (E) PARP were also increased when MTF (8 mM) was added. The results are expressed as the mean \pm standard deviation for experiments performed in triplicate. ${ }^{* *} \mathrm{P}<0.01$ and ${ }^{* * *} \mathrm{P}<0.001$. NDP, nedaplatin; $\mathrm{MTF}$, metformin.

The main adverse effect of platinum is myelosuppression (20), so if MTF can improve chemotherapy sensitivity, it will help to decrease the dosage of NDP to achieve a similar killing effect to the regimens without MTF (12). However, it is not certain whether all patients with ovarian cancer may benefit from this drug at present, as cases of MTF resistance have been reported $(30,31)$. For example, in vitro cell experiments, in the ovarian cancer cell line SKOV3ip were reported to be not as sensitive to MTF as other ovarian cancer cells (30).

In clinical practice, identifying patients who would benefit the most from the use of MTF in combination chemotherapy is the top priority. However, the lack of clinical indicators and tests for rapid prediction has become a major constraint to the clinical application of MTF in the treatment of ovarian cancer $(32,33)$. Great efforts have been made to elucidate the mechanism of action of MTF. A previous study indicated that MTF downregulated the expression of the anti-apoptotic proteins B-cell lymphoma 2 (Bcl-2) and myeloid cell leukaemia 1, and upregulated the expression of the pro-apoptotic protein $\mathrm{Bcl}$-2-associated $\mathrm{X}$ protein (Bax) in cancer cells $(34,35)$. It has been suggested that MTF may serve an antitumor role directly via an AMP-dependent protein kinase (AMPK)-dependent or AMPK-independent pathway (31). In addition, MTF may increase the transcriptional expression of p53 and its downstream targets Bax and $\mathrm{p} 21$, thus promoting apoptosis and inhibiting tumor growth (36). A previous study demonstrated that MTF blocked cancer cells in the G1 stage (37) and induced cell cycle stagnation or apoptosis. Cancer stem cells appear to be key to chemoresistance and tumor relapse. A previous study implied that MTF at a low dose selectively inhibited $\mathrm{CD} 44^{+} \mathrm{CD} 117^{+}$ovarian cancer stem cells through inhibition of epithelial-mesenchymal transition and potentiated the effect of DDP (38). Although in numerous studies, the dosages of MTF were higher compared with the dosage in clinical patients, the use of MTF has increased the understanding of the mechanisms of tumor cell chemoresistance, metastasis and recurrence (39-41). However, there was no consensus in these studies concerning the markers to identify patients that would benefit from MTF. MTF was traditionally applied in patients with type 2 diabetes 
mellitus; however, there is not enough evidence to support its use in all patients without type 2 diabetes mellitus, and MTF-resistant cells have also been identified $(30,31)$. The method developed in the present study may help to identify patients sensitive to MTF. However, as the present study only concerned one case, an increased number of cases including patients with type 2 diabetes mellitus will be recruited for further study. In conclusion, the sensitivity of OM cells to different platinum-based regimens varied considerably. The real-time cell analyzer assay may assist in selecting personalized chemotherapy regimens in clinical practice. Investigating and clarifying the underlying molecular mechanism of MTF, a drug with a chemotherapy sensitization effect, may shed some light on the treatment of ovarian cancers, particularly those with extensive metastatic tumors.

\section{Acknowledgements}

Not applicable.

\section{Funding}

The present study was supported by the National Natural Science Foundation of China (grant no. 81360341), National Natural Science Foundation of China (grant no. 81560428), National High Technology Research and Development Program (863 Program) (grant no. 2014AA020605), Guangxi Zhuang Autonomous Region Health and Family Planning Commission Self-Financing Project (grant no. Z2015578) and Guangxi Nanning Qingxiu District Science and Technology Development Project (grant no. 2015S14).

\section{Availability of data and materials}

The datasets used and/or analyzed during the current study are available from the corresponding author on reasonable request.

\section{Authors' contributions}

YT, QW and YF contributed to the conception and design of the work. YL, YF, JW and HL contributed to the acquisition, analysis and interpretation of data. YF and YL drafted the manuscript. QW and YT revised the manuscript and gave final approval of the version to be published.

\section{Ethics approval and consent to participate}

Written informed consent for this research was obtained from the patient prior to surgery. The ethics review committee of The Affiliated Tumor Hospital of Guangxi Medical University approved the present study.

\section{Patient consent for publication}

The patient has reviewed the material to be published and provided written permission for the publication.

\section{Competing interests}

The authors declared that they have no competing interests.

\section{References}

1. Coleman RL: Ovarian cancer in 2015: Insights into strategies for optimizing ovarian cancer care. Nat Rev Clin Oncol 13: 71-72, 2016.

2. Metzger-Filho O, Moulin C and D'Hondt V: First-line systemic treatment of ovarian cancer: A critical review of available evidence and expectations for future directions. Curr Opin Oncol 22: 513-520, 2010.

3. Kipps E, Tan DS and Kaye SB: Meeting the challenge of ascites in ovarian cancer: New avenues for therapy and research. Nat Rev Cancer 13: 273-282, 2013.

4. Roshan Moniri M, Young A, Reinheimer K, Rayat J, Dai LJ and Warnock GL: Dynamic assessment of cell viability, proliferation and migration using real time cell analyzer system (RTCA). Cytotechnology 67: 379-386, 2015.

5. Pan T, Huang B, Zhang W, Gabos S, Huang DY and Devendran V: Cytotoxicity assessment based on the AUC50 using multi-concentration time-dependent cellular response curves. Anal Chim Acta 764: 44-52, 2013

6. Kho D, MacDonald C, Johnson R, Unsworth CP, O'Carroll SJ, du Mez E, Angel CE and Graham ES: Application of xCELLigence RTCA biosensor technology for revealing the profile and window of drug responsiveness in real time. Biosensors (Basel) 5: 199-222, 2015.

7. Bailey CJ: Metformin: Historical overview. Diabetologia 60: 1566-1576, 2017.

8. Decensi A, Puntoni M, Goodwin P, Cazzaniga M, Gennari A, Bonanni B and Gandini S: Metformin and cancer risk in diabetic patients: A systematic review and meta-analysis. Cancer Prev Res (Phila) 3: 1451-1461, 2010.

9. Romero IL, McCormick A, McEwen KA, Park S, Karrison T, Yamada SD, Pannain S and Lengyel E: Relationship of type II diabetes and metformin use to ovarian cancer progression, survival, and chemosensitivity. Obstet Gynecol 119: 61-67, 2012.

10. Kumar S, Meuter A, Thapa P, Langstraat C, Giri S, Chien J, Rattan R, Cliby W and Shridhar V: Metformin intake is associated with better survival in ovarian cancer: A case-control study. Cancer 119: 555-562, 2013.

11. Bar D, Lavie O, Stein N, Feferkorn I and Shai A: The effect of metabolic comorbidities and commonly used drugs on the prognosis of patients with ovarian cancer. Eur J Obstet Gynecol Reprod Biol 207: 227-231, 2016.

12. Patel S, Singh N and Kumar L: Evaluation of effects of metformin in primary ovarian cancer cells. Asian Pac J Cancer Prev 16: 6973-6979, 2015.

13. Mutch DG and Prat J: 2014 FIGO staging for ovarian, fallopian tube and peritoneal cancer. Gynecol Oncol 133: 401-404, 2014.

14. Metzgerfilho O, Moulin C and D'hondt V: First-line systemic treatment of ovarian cancer: A critical review of available evidence and expectations for future directions. Curr Opin Oncol 22: 513-520, 2010.

15. Siegel RL, Miller KD and Jemal A: Cancer statistics, 2017. CA Cancer J Clin 67: 7-30, 2017.

16. Howlader N, Noone AM, Krapcho M, Miller D, Bishop K, Kosary CL, Yu M, Ruhl J, Tatalovich Z, Mariotto A, et al (eds): SEER cancer statistics review, 1975-2014, National Cancer Institute. Bethesda MD, https://seer.cancer.gov/csr/1975_2014/, based on November 2016 SEER data submission, posted to the SEER web site, April, 2017.

17. Mei L, Chen H, Wei DM, Fang F, Liu GJ, Xie HY, Wang X, Zou J, Han X and Feng D: Maintenance chemotherapy for ovarian cancer. Cochrane Database Syst Rev 29: CD007414, 2013.

18. Davidson B and Tropé CG: Ovarian cancer: Diagnostic, biological and prognostic aspects. Womens Health (Lond) 10: 519-533, 2014

19. Holmes D: Ovarian cancer: Beyond resistance. Nature 527: S217, 2015.

20. Matulonis UA, Sood AK, Fallowfield L, Howitt BE, Sehouli J and Karlan BY: Ovarian cancer. Nat Rev Dis Primers 2: 16061, 2016.

21. Kischkel FC, Meyer C, Eich J, Nassir M, Mentze M, Braicu I, Kopp-Schneider A and Sehouli J: Prediction of clinical response to drugs in ovarian cancer using the chemotherapy resistance test (CTR-test). J Ovarian Res 10: 72, 2017.

22. O Donnell RL, Mccormick A, Mukhopadhyay A, Woodhouse LC, Moat M, Grundy A, Dixon M, Kaufman A, Soohoo S, Elattar A, et al: The use of ovarian cancer cells from patients undergoing surgery to generate primary cultures capable of undergoing functional analysis. PLoS One 9: e90604, 2014. 
23. Kar R, Chawla D, Gupta B, Mehndiratta M, Wadhwa N and Agarwal R: Establishment of primary cell culture from ascitic fluid and solid tumor obtained from epithelial ovarian carcinoma patients. Int J Gynecol Cancer 27: 2000-2005, 2017.

24. Robinson DR, Wu YM, Lonigro RJ, Vats P, Cobain E, Everett J, Cao X, Rabban E, Kumar-Sinha C, Raymond V, et al: Integrative clinical genomics of metastatic cancer. Nature 548: 297-303, 2017.

25. Gillet JP and Gottesman MM: Mechanisms of multidrug resistance in cancer. Methods Mol Biol 596: 47-76, 2010.

26. Sherman-Baust CA, Becker KG, Wood Iii WH, Zhang Y and Morin PJ: Gene expression and pathway analysis of ovarian cancer cells selected for resistance to cisplatin, paclitaxel, or doxorubicin. J Ovarian Res 4: 21, 2011.

27. Martin LP, Hamilton TC and Schilder RJ: Platinum resistance: The role of DNA repair pathways. Clin Cancer Res 14: 1291-1295, 2008.

28. Shen D, Pouliot LM, Hall MD and Gottesman MM: Cisplatin resistance: A cellular self-defense mechanism resulting from multiple epigenetic and genetic changes. Pharmacol Rev 64: 706-721, 2012.

29. Sato $\mathrm{S}$ and Itamochi $\mathrm{H}$ : Ovarian cancer and drug resistance. Curr Obstet Gynecol Rep 4: 18-25, 2015.

30. Rattan R, Giri S, Hartmann LC and Shridhar V: Metformin attenuates ovarian cancer cell growth in an AMP-kinase dispensable manner. J Cell Mol Med 15: 166-178, 2011.

31. Do MT, Kim HG, Choi JH and Jeong HG: Metformin induces microRNA-34a to downregulate the Sirt1/Pgc-1 $\alpha / \mathrm{Nrf} 2$ pathway, leading to increased susceptibility of wild-type p53 cancer cells to oxidative stress and therapeutic agents. Free Radic Biol Med 74: 21-34, 2014.

32. Shank JJ, Yang K, Ghannam J, Cabrera L, Johnston CJ, Reynolds RK and Buckanovich RJ: Metformin targets ovarian cancer stem cells in vitro and in vivo. Gynecol Oncol 127: 390-397, 2012.

33. Romero IL, McCormick A, McEwen KA, Park S, Karrison T, Yamada SD, Pannain S and Lengyel E: Relationship of type II diabetes and metformin use to ovarian cancer progression, survival, and chemosensitivity. Obstet Gynecol 119: 61-67, 2012.
34. Gwak H, Kim Y, An H, Dhanasekaran DN and Song YS: Metformin induces degradation of cyclin D1 via AMPK/GSK3 $\beta$ axis in ovarian cancer. Mol Carcinog 56: 349-358, 2017.

35. Wang H, Zhang Z, Wei X and Dai R: Small-molecule inhibitor of $\mathrm{Bcl}-2$ (TW-37) suppresses growth and enhances cisplatin-induced apoptosis in ovarian cancer cells. J Ovarian Res 8: 3, 2015.

36. Li P, Zhao M, Parris AB, Feng X and Yang X: p53 is required for metformin-induced growth inhibition, senescence and apoptosis in breast cancer cells. Biochem Biophys Res Commun 464: 1267-1274, 2015

37. Gao ZY, Liu Z, Bi MH, Zhang JJ, Han ZQ, Han X, Wang HY, Sun GP and Liu H: Metformin induces apoptosis via a mitochondria-mediated pathway in human breast cancer cells in vitro. Exp Ther Med 11: 1700-1706, 2016.

38. Zhang R, Zhang P, Wang H, Hou D, Li W, Xiao G and Li C: Inhibitory effects of metformin at low concentration on epithelial-mesenchymal transition of CD44(+)CD117(+) ovarian cancer stem cells. Stem Cell Res Ther 6: 262, 2015.

39. Gonzalez DM and Medici D: Signaling mechanisms of the epithelial-mesenchymal transition. Sci Signal 7: re8, 2014.

40. Ling S, Song L, Fan N, Feng T, Liu L, Yang X, Wang M, Li Y, Tian Y, Zhao F, et al: Combination of metformin and sorafenib suppresses proliferation and induces autophagy of hepatocellular carcinoma via targeting the mTOR pathway. Int J Oncol 50: 297-309, 2017.

41. Chou CC, Lee KH, Lai IL, Wang D, Mo X, Kulp SK, Shapiro CL and Chen CS: AMPK reverses the mesenchymal phenotype of cancer cells by targeting the Akt-MDM2-Foxo3a signaling axis Cancer research 74: 4783-4795, 2014.

This work is licensed under a Creative Commons Attribution-NonCommercial-NoDerivatives 4.0 International (CC BY-NC-ND 4.0) License. 